\title{
Can biopsy be avoided in patients with clinical suspicion of prostate cancer and a negative result on multiparametric magnetic resonance imaging?
}

\author{
Ronaldo Hueb Baroni ${ }^{1}$
}

Multiparametric magnetic resonance imaging (mpMRI) of the prostate, given its good spatial resolution and excellent contrast resolution, has been used for many years in the local staging of prostate cancer prior to treatment ${ }^{(\mathbf{1})}$. More recently, especially after the development of the standardized criteria of Prostate Imaging Reporting and Data System (PI-RADS) version $2^{(2)}$, mpMRI was also incorporated into the strategies for prostate cancer screening/detection. In patients with elevated serum prostatic-specific antigen (PSA) or altered digital rectal examination results, many centers now recommend an mpMRI scan before transrectal ultrasound-guided biopsy, in order to detect suspicious foci for clinically significant prostate cancer (csPCa)-those with a Gleason score $>6$ or International Society of Urological Pathology group $>1$-and increase the likelihood of a positive targeted biopsy. Many articles published in the most prestigious scientific journals, such as those conducted by the multicenter study-groups "PROMIS" and "PRECISION"(3,4), have shown that performing a targeted biopsy after a positive mpMRI increases the detection of csPCa and decreases the detection of tumors that are not clinically significant. A recent systematic review and metaanalysis confirmed the superiority of targeted biopsy over systematic biopsy ${ }^{(5)}$. On the basis of those data, the European Association of Urology guidelines now recommend performing mpMRI prior to biopsy, not only for biopsy-naïve patients but also for those with a prior negative biopsy ${ }^{(6)}$. However, the negative predictive value (NPV) of a negative mpMRI result (PI-RADS 1 or 2 ) in patients with suspected prostate cancer is less well established. A meta-analysis published in 2015 showed that the pooled-data NPV of mpMRI using PI-RADS version 1 to detect cancer ranged from 58\% to 95\%, although there was a lot of variability in the interpretation of PI-RADS findings among the studies analyzed ${ }^{(7)}$.

In general, we can divide studies focusing on the NPV of mpMRI into two groups: those that evaluated the results of

1. Medical Coordinator of the Magnetic Resonance Section and Head of the Abdominal Imaging Group at Hospital Israelita Albert Einstein, São Paulo, SP, Brazil. Email: rbaroni@einstein.br. https://orcid.org/0000-0001-8762-0875. systematic biopsies in patients with a negative mpMRI result; and those that followed patients with a negative mpMRI result for a period of time (using PSA, subsequent mpMRI scans, and, in some cases, biopsies to confirm the true negativity of the initial imaging study). In a study conducted in 2016, Hansen et al. ${ }^{(\mathbf{8})}$ performed transperineal targeted and saturation biopsies after a negative initial mpMRI result (using PI-RADS version 1), reporting an NPV of $92 \%$. In the multicenter PROMIS study, patients with a negative result on a $1.5 \mathrm{~T}$ mpMRI scan underwent template transperineal biopsy, with an NPV of $76 \%{ }^{(3)}$. From another perspective, a well-designed study conducted by Itatani et al. ${ }^{(9)}$ followed patients with an initial negative mpMRI result for 5 years, reporting an NPV of $89.6 \%$ for csPCa. The most important article focusing on the NPV of mpMRI might be the systematic review and meta-analysis conducted by Moldovan et al. ${ }^{(\mathbf{1 0})}$ in 2017 , in which the median NPV of the selected studies was $88.1 \%$ for csPCa. The authors also reporting that the NPV decreases significantly when the prevalence of cancer increases ${ }^{(\mathbf{1 0})}$.

The very interesting article authored by Baghdanian et al. ${ }^{(11)}$, published in this issue of Radiologia Brasileira, focuses on the differences in the NPV of prostate mpMRI between men with suspected cancer and those with known cancer. They found that the NPV for csPCa was $87.2 \%$ in men with suspected cancer and $64.1 \%$ in men under active surveillance. Their study underscores the fact that the variability of the NPV of mpMRI in the literature is related, at least to some extent, to the differences in cancer prevalence among the different study populations. In a study published in 2018, Panebianco et al. ${ }^{\text {(12) }}$ found that the probability of disease-free survival after a negative mpMRI result was significantly higher in patients with a prior negative biopsy than in biopsy-naïve patients (96\% vs. 84\%).

It is fair to say that a negative mpMRI result, especially in patients with a low pre-test probability for prostate cancer (e.g., those with a PSA < 0.15), is a reliable indicator to avoid a systematic biopsy, at least initially. The European Association of Urology has already incorporated this recommendation in their guidelines ${ }^{(6)}$. 


\section{REFERENCES}

1. Wang L, Hricak H, Kattan MW, et al. Prediction of organ-confined prostate cancer: incremental value of MR imaging and MR spectroscopic imaging to staging nomograms. Radiology. 2006;238:597-603.

2. Weinreb JC, Barentsz JO, Choyke PL, et al. PI-RADS Prostate Imaging - Reporting and Data System: 2015, Version 2. Eur Urol. 2016;69:16-40.

3. Ahmed HU, El-Shater Bosaily A, Brown LC, et al. Diagnostic accuracy of multiparametric MRI and TRUS biopsy in prostate cancer (PROMIS): a paired validating confirmatory study. Lancet. 2017;389:815-22.

4. Kasivisvanathan V, Rannikko AS, Borghi M, et al. MRI-targeted or standard biopsy for prostate-cancer diagnosis. N Engl J Med. 2018;378:1767-77.

5. KasivisvanathanV, Stabile A, Neves JB, et al. Magnetic resonance imaging-targeted biopsy versus systematic biopsy in the detection of prostate cancer: a systematic review and meta-analysis. Eur Urol. 2019;76:284-303.

6. European Association of Urology. Prostate cancer guidelines 2019. Available from: https://uroweb.org/guideline/prostate-cancer.

7. Hamoen EHJ, de Rooij M, Witjes JA, et al. Use of the Prostate Imaging Reporting and Data System (PI-RADS) for prostate cancer detection with multipara- metric magnetic resonance imaging: a diagnostic meta-analysis. Eur Urol. 2015;67:1112-21.

8. Hansen NL, Kesch C, Barrett T, et al. Multicentre evaluation of targeted and systematic biopsies using magnetic resonance and ultrasound image-fusion guided transperineal prostate biopsy in patients with a previous negative biopsy. BJU Int. 2017;120:631-8.

9. Itatani R, Namimoto T, Atsuji S, et al. Negative predictive value of multiparametric MRI for prostate cancer detection: outcome of 5-year follow-up in men with negative findings on initial MRI studies. Eur J Radiol. 2014;83:1740-5.

10. Moldovan PC, Van den Broeck T, Sylvester R, et al. What is the negative predictive value of multiparametric magnetic resonance imaging in excluding prostate cancer at biopsy? A systematic review and meta-analysis from the European Association of Urology Prostate Cancer Guidelines Panel. Eur Urol. 2017;72:250-66.

11. Baghdanian AA, Kim YJ, Baghdanian AH, et al. Differences in negative predictive value of prostate MRI based in men with suspected or known cancer. Radiol Bras. 2019;52:281-6.

12. Panebianco V, Barchetti G, Simone G, et al. Negative multiparametric magnetic resonance imaging for prostate cancer: what's next? Eur Urol. 2018;74:48-54.

\section{$(c))$ BY}

